\title{
Reforma local, servicios sociales y perspectiva de género
}

\author{
Elena ROLDÁN GARCÍA ${ }^{1}$ \\ Facultad de Trabajo Social. Universidad Complutense de Madrid \\ eroldang@ucm.es \\ Begoña LEYRA FATOU ${ }^{2}$ \\ Facultad de Trabajo Social. Universidad Complutense de Madrid \\ mbleyra@ucm.es
}

Recibido: 01.09.2014

Aceptado: 22.01.2015

\section{RESUMEN:}

El nuevo marco legal para las administraciones locales, la Ley 27/2013 de 27 de diciembre, de racionalización y sostenibilidad de la Administración Local (LRSAL) modifica substancialmente su ámbito competencial y, afecta de manera específica a los servicios sociales y a la perspectiva de género. Tras la entrada en vigor de esta normativa, este artículo aporta algunas claves interpretativas para determinar, en primer lugar, cómo ha sido en etapas precedentes el proceso de institucionalización de las políticas locales de servicios sociales, la incorporación de la perspectiva de género y las reformas que se han ido produciendo. En segundo lugar se abordan sintéticamente los principales marcos de referencia de la estructuración de los servicios sociales y de las políticas de género: cohesión social, desigualdad, transversalidad e interseccionalidad. A continuación se realiza un examen crítico de la nueva legislación y su impacto en los servicios sociales municipales. Se concluye con una valoración de las posibilidades e incertidumbres del futuro de los servicios sociales y de la perspectiva de género tras esta reforma local. Somos conscientes de que la aprobación tan reciente de esta nueva legislación no permite todavía un análisis pormenorizado de su aplicación y sus consecuencias, sin embargo, se pueden vislumbrar diferentes opciones de cara al futuro que constituyen elementos para el debate, como qué tipo de decisiones habrá que tomar en aras de paliar las incidencias negativas sobre los servicios sociales y la perspectiva de género de esta normativa, muy criticada por sectores profesionales y académicos que no dudan algunos en calificarla de imprecisa, contradictoria e incompleta.

1 Datos de contacto: Universidad Complutense de Madrid. Facultad de Trabajo Social. Departamento de Trabajo Social y Servicios Sociales. Despacho 2106. Teléfono 913942817. Correo electrónico: eroldang@ucm.es

2 Datos de contacto: Universidad Complutense de Madrid. Facultad de Trabajo Social. Departamento de Trabajo Social y Servicios Sociales. Despacho 2113. Teléfono 913942730. Correo electrónico: mbleyra@ucm.es 
Palabras clave: Políticas de Servicios Sociales; Reforma legislativa local; Perspectiva de género; Cohesión social.

\title{
Local government reform, social services and gender perspective
}

\begin{abstract}
:
The new legal framework for local government, the Law 27/2013 of 27 December, on Rationalization and Sustainability of Local Government (LRSAL) substantially modifies its powers and, specifically, in social services and gender perspective. Following the entry into force of this regulation, this paper provides some interpretive keys to determine, first, how it has been in previous stages the institutionalization of local social services policies, the incorporation of a gender perspective and reforms which have taken place. Secondly, it addresses briefly, the main theoretical frameworks of structuring social services and gender policies: social cohesion, inequality, mainstreaming and intersectionality. Subsequently, a critical review of the new legislation and its impact on social services is performed. It concludes with an assessment of the possibilities and uncertainties of the future of social services and gender perspective after this local reform. We are aware that the recent approval of this new legislation does not yet allow a detailed analysis of its application and its consequences, however, can be glimpsed different options to the future which constitute elements for the debate as: what kind of decisions will have to be taken in order to mitigate the negative impact on social services and gender perspective of this law, criticized by professionals and academics who do not hesitate to describe it as some vague, contradictory and incomplete.
\end{abstract}

Keywords: Social Services Policies; Local legislative reform; Gender perspective; Social cohesion.

\section{INTRODUCCIÓN}

Este trabajo aporta algunas claves interpretativas para determinar, tras la entrada en vigor de la Ley 27/2013 de 27 de diciembre, de racionalización y sostenibilidad de la Administración Local (LRSAL), por una parte, cómo ha sido el proceso de institucionalización de las políticas locales de servicios sociales y las reformas que se han producido en las últimas décadas, y por la otra la incorporación de la perspectiva de género en este ámbito. En segundo lugar se abordan sintéticamente los principales marcos de referencia de la implantación de los servicios sociales en general y de forma específica aquellos que han constituido la base de la estructuración de las políticas de género en este campo: cohesión social, desigualdad, transversalidad e interseccionalidad. A continuación, en tercer lugar, se realiza un análisis de la nueva legislación y su impacto en los servicios sociales y en la perspectiva de género, partiendo de un examen crítico sobre las modificaciones que la LRSAL realiza de las competencias de los Ayuntamientos establecidas en la Ley Reguladora de las Bases de Régimen Local de 1985 (LRBRL) y en concreto, cómo afecta a los servicios sociales municipales. Finalmente, y a modo de conclusiones, se lleva a cabo una valoración de las posibilidades e incertidumbres del futuro de los servicios sociales locales y la 
perspectiva género tras la aplicación de la nueva normativa. Conscientes de que la reciente aprobación de esta legislación no permite todavía un análisis pormenorizado de su aplicación y sus consecuencias, sin embargo, se pueden vislumbrar diferentes opciones de cara al futuro que constituyen elementos para el debate, como son las decisiones por las qué habrá que optar en aras de paliar las posibles incidencias negativas sobre los servicios sociales y la perspectiva género de esta normativa, muy criticada por sectores profesionales y académicos que no dudan algunos en calificarla de impreciso, contradictorio e incompleto.

\section{POLÍTICAS LOCALES DE SERVICIOS SOCIALES: INSTITUCIONALIZACIÓN, LÍMITES Y PERSPECTIVA DE GÉNERO}

Desde hace cerca de dos décadas se viene debatiendo sobre la necesidad de una nueva legislación de régimen local capaz de dar respuestas a los diversos y complejos problemas que presenta la Administración local. Sin embargo, el hecho de que se haya aprobado la nueva Ley 27/2013 de 27 de diciembre, de racionalización y sostenibilidad de la Administración Local (LRSAL) no parece que haya solventado las dificultades que presenta este nivel de la Administración, ya que la nueva normativa está recibiendo importantes críticas por parte de los sectores profesionales y académicos. Si bien esta normativa se enmarca en una amplia política gubernamental de reforma de la Administración española con valoraciones positivas por parte de organismos internacionales (OECD, 2014).

Antes de entrar a analizar de forma pormenorizada su impacto en las políticas locales de servicios sociales desde la perspectiva de género, es preciso llevar a cabo un breve recorrido por los avatares normativos del ámbito local y su incidencia en el desarrollo de las políticas locales de servicios sociales. La Constitución española de 1978 no estableció unas competencias concretas para los entes locales, sino que se limitó a reconocerles autonomía en la gestión de sus respectivos intereses (arts.137 y 140). La concreción de este principio constitucional y su desarrollo por los consistorios se expresó en la Ley 7/85 Reguladora de las Bases del Régimen Local (LRBRL). Esta ley estableció la competencia municipal en la prestación de servicios sociales de forma muy genérica, aunque con carácter obligatorio en los municipios mayores de 20.000 habitantes (artículos 25.2.k) y 26.1.c)) pudiendo ejercerla solos o asociados con otros ayuntamientos. La realidad española presenta el hecho de que la mayor parte de sus municipios tienen una población inferior a los 20.000 habitantes, por lo tanto cerca del $80 \%$ de los mismos no se habrían encontrado obligados legalmente a prestar servicios sociales. No obstante, el proceso histórico del desarrollo de los primeros ayuntamientos democráticos, indica que sus políticas públicas fueron cubriendo el déficit y las enormes carencias que en esta materia se arrastraban. A pesar de la falta de concreción en las competencias directas en servicios sociales de 
los municipios, se fue dando respuesta a las demandas más o menos inmediatas de la ciudadanía por ser su estructura más cercana (Roldán y García, 2006; Subirats, 2007).

El hecho de que en 1988 España se adhiriera a la Carta Europea de la Autonomía Local ${ }^{3}$, supuso un impulso al desarrollo de políticas en materia de servicios sociales en el ámbito local. La indicada Carta expone en su preámbulo que "la defensa y el fortalecimiento de la autonomía local representan una contribución especial en la construcción de una Europa basada en los principios de democracia y descentralización del poder". La autonomía local se define como "el derecho y la capacidad efectiva de las Entidades Locales de ordenar y gestionar una parte importante de los asuntos públicos, en el marco de la Ley, bajo su propia responsabilidad y en beneficio de sus habitantes" (art. 3.1); y se señala además que el "ejercicio de las responsabilidades públicas debe, de modo general, incumbir preferentemente a las autoridades más cercanas a los ciudadanos" (art. 4.3).

Los municipios fueron asumiendo así el liderazgo local y sus responsables construyeron un modelo de gobierno local omnicomprensivo, no tanto por voluntad propia, sino por la misma dinámica de respuesta a las demandas indiferenciadas $\mathrm{y}$ urgentes de la población. Pero las limitaciones de las propias estructuras municipales españolas provenientes de los rasgos peculiares de la política y de la Administración local han venido dando cuenta de la necesidad de una reforma local en profundidad. Por una parte, se ha desarrollado un tipo de gobierno local muy "presidencialista" con un gran poder del alcalde/sa en la implantación y desarrollo de las políticas públicas, con oportunidades para abuso de poder tanto personal como partidista, junto a ello, altos cargos y personal funcionario de la Administración local, poseen un margen restringido de actuaciones, limitado básicamente a un control legalista (Lapuente, 2014).

El debate sobre la reforma local ha dado lugar a tímidas actuaciones y marcos normativos. Desde el punto de vista estructural o de modelo, lo más significativo ha sido la reforma de 1999 que surge como consecuencia del denominado "Pacto Local" y la reforma de 2003. Así, la Ley 11/1999, de 21 de abril $^{4}$ se limitó a favorecer un modelo de gobierno presidencialista con

${ }^{3}$ Recuperado en: http:/www.seap.minhap.es/dms/es/areas/politica_local/participacion-eellaeuropeos/cartaeuropea.pdf (Junio, 28, 2014).

${ }^{4}$ Ley 11/1999, de 21 de abril, de modificación de la Ley 7/1985, de 2 de abril, Reguladora de las Bases del Régimen Local, y otras medidas para el desarrollo del Gobierno Local, en materia de tráfico, circulación de vehículos a motor y seguridad vial y en materia de aguas. Recuperado en: http://www.boe.es/boe/dias/1999/04/22/pdfs/A14928-14936.pdf (Junio, 20, 2014). 
fortalecimiento de la figura del alcalde/sa, sin establecer un régimen especial para las grandes ciudades. La reforma introducida por la Ley 57/2003, de 16 de diciembre, de medidas para la modernización del gobierno local ${ }^{5}$ fue consciente de su insuficiencia desde su aprobación, por lo que precisamente fijó como objetivo último "la elaboración de una nueva Ley de Bases de la Administración Local, que constituya un instrumento adecuado para que nuestros gobiernos locales afronten los complejos retos que les presentan los albores del siglo XXI". Posteriormente se redactaron el Libro Blanco de la Reforma del Gobierno Local de $2005^{6}$ y el Borrador de Anteproyecto de Ley de Bases del Gobierno Local. Aprobados los Estatutos de autonomía de segunda generación, el Anteproyecto de Ley de Bases del Gobierno Local "acabó en un cajón”.

En este marco ha tenido lugar el desarrollo del sistema público de servicios sociales de base fundamentalmente municipalista, pero sustentado por las Comunidades Autónomas (CC.AA.) las cuales, han legislado sobre esta materia desde 1982. Las renovaciones legislativas y la aprobación de la Ley 39/2006 de promoción de la autonomía personal y atención a las situaciones de dependencia (LAPAD), han intentado afianzar el sistema, pero las dificultades en la consolidación del mismo tienen relación directa con la situación social y económica de los últimos años y con el proceso de los cambios democráticos a partir de la Constitución de 1978 y las limitaciones del sistema benéfico-asistencial precedente. Asimismo, este proceso se ha visto debilitado por la incidencia de los recortes en protección social que se están llevando a cabo a partir de las políticas neoliberales y que se han agudizado con la crisis económica y financiera de 2008. De este modo, no se ha logrado alcanzar un lugar en el conjunto del sistema de protección social y los servicios sociales continúan en una posición relegada y benéfico-asistencial en el mundo de "los pobres y de los grupos de riesgo" (Santamarina, Mendiguren, Mondine y Roldán, 1999; Adelantado y Jiménez, 2003; Rodríguez Cabrero, 2004a; Aguilar, 2010; Antón, 2012; Roldán, García y Nogués, 2013), aspecto este que puede reforzarse con la nueva Ley 27/2013 de 27 de diciembre, de racionalización y sostenibilidad de la Administración Local (LRSAL) como se demostrará a lo largo de los próximos epígrafes.

Tabla 1: Principal marco normativo anterior a la LRSAL

${ }^{5}$ Recuperado en: http://www.boe.es/boe/dias/2003/12/17/pdfs/A44771-44791.pdf (Junio, 20, 2014).

${ }^{6}$ Recuperado en:

http://www.seap.minhap.gob.es/dms/es/publicaciones/centro de_publicaciones_de la_sgt/ Monografias/parrafo/0111111111111111118/text es files/Libro-Blanco-GobiernoLocal.pdf (Junio, 25, 2014). 


\begin{tabular}{|l|c|l|}
\hline \multicolumn{1}{|c|}{ Marco normativo } & Fecha & \multicolumn{1}{|c|}{ Referencias significativas } \\
\hline Constitución Española & 1978 & $\begin{array}{l}\text { Reconocimiento de la autonomía en la gestión de los } \\
\text { intereses de los entes locales (arts.137 y 140). }\end{array}$ \\
\hline $\begin{array}{l}\text { Ley 7/85 Reguladora de } \\
\text { las Bases del Régimen } \\
\text { Local }\end{array}$ & 1985 & $\begin{array}{l}\text { Competencia municipal en la prestación de servicios } \\
\text { sociales y con carácter obligatorio en los municipios } \\
\text { mayores de 20.000 habitantes (artículos 25.2.k) y 26.1.c)) }\end{array}$ \\
\hline $\begin{array}{l}\text { Carta Europea de la } \\
\text { Autonomía Local }\end{array}$ & 1985 & $\begin{array}{l}\text { La autonomía local: "el derecho y la capacidad efectiva de } \\
\text { las Entidades Locales de ordenar y gestionar una parte } \\
\text { importante de los asuntos públicos, en el marco de la Ley, } \\
\text { bajo su propia responsabilidad y en beneficio de sus } \\
\text { habitantes" (art. 3.1);"El ejercicio de las responsabilidades } \\
\text { públicas debe, de modo general, incumbir preferentemente a } \\
\text { las autoridades más cercanas a los ciudadanos" (art. 4.3). }\end{array}$ \\
\hline $\begin{array}{l}\text { Ley 11/1999, de 21 de } \\
\text { abril }\end{array}$ & 1999 & $\begin{array}{l}\text { Favorece un modelo de gobierno presidencialista con } \\
\text { fortalecimiento de la figura del alcalde/sa }\end{array}$ \\
\hline $\begin{array}{l}\text { Ley 57/2003, de 16 de } \\
\text { diciembre, de medidas } \\
\text { para la modernización del } \\
\text { gobierno local }\end{array}$ & 2003 & $\begin{array}{l}\text { Fijó como objetivo último "la elaboración de una nueva Ley } \\
\text { de Bases de la Administración Local, que constituya un } \\
\text { instrumento adecuado para que nuestros gobiernos locales } \\
\text { afronten los complejos retos que les presentan los albores } \\
\text { del siglo XXI". }\end{array}$ \\
\hline $\begin{array}{l}\text { Libro Blanco de la } \\
\text { Reforma del Gobierno } \\
\text { Local de 2005 }\end{array}$ & 2005 & $\begin{array}{l}\text { "Inicio del proceso de redacción del proyecto de Ley sobre } \\
\text { el Gobierno y la Administración Local" (Prólogo) }\end{array}$ \\
\hline $\begin{array}{l}\text { Borrador de Anteproyecto } \\
\text { de Ley de Bases del } \\
\text { Gobierno Local }\end{array}$ & 2006 & $\begin{array}{l}\text { Listado de materias sobre las que los municipios ostentan } \\
\text { competencias para la ordenación y prestación de servicios } \\
\text { básicos locales. } \\
\text { prosperó) } \\
\text { Fijación de un elenco de materias sobre las que la } \\
\text { legislación sectorial estatal y autonómica garanticen la } \\
\text { intervención municipal }\end{array}$ \\
\hline
\end{tabular}

Fuente: Elaboración propia 
Los límites del sistema provienen de la propia complejidad de la noción de servicios sociales y de las dificultades para establecer una definición de los mismos (Rodríguez Cabrero, 2004b; Aguilar, 2010; Gómez Alguacil, 2012; Roldán, García y Nogués, 2013). Sintéticamente se puede apuntar que comprenden prestaciones con la finalidad de garantizar la dignidad de la persona, su desarrollo pleno y autonomía en la sociedad; para promover su participación en la vida ciudadana y conseguir prevenir, tratar o paliar las situaciones de riesgo de exclusión social, estimulando capacidades y actitudes de relación interpersonal e integración social. De este modo la vulnerabilidad viene siendo un elemento definitorio de la actuación de los servicios sociales y entre los grupos vulnerables se ha tomado en consideración a las mujeres, por lo que se han configurado como un grupo esencial en la actuación de los servicios sociales. Un análisis de las razones por las que se ha producido esta situación permitirá examinar posteriormente las estrategias políticas que se han ido desarrollando para superar la misma. Las mujeres (al igual que niños y niñas), históricamente, han sido imaginadas erróneamente como un colectivo vulnerable, enmarcado en las consideraciones de la pobreza femenina, en el que esta se analizaba como un fenómeno unilateral (basado en el ingreso), sin cuestionar las causas de la pobreza y la desigualdad, y sin conceder a las mujeres un rol de ciudadanas con derechos que pueden ser agentes de cambio de su propia condición. Por ello, hay que tomar en consideración tres conceptos que explican la incorporación de las mujeres como grupo predominante en los servicios sociales: la vulnerabilidad, la exclusión y la desigualdad (Clert, 1998 y Kabeer, 1998 y 2003):

- $\quad$ El enfoque de vulnerabilidad surge con fuerza a fines de los años ochenta, y contribuye a iluminar algunos procesos que llevan a la pobreza de las mujeres. Tiene dos facetas, una externa que se refiere a los riesgos a los que un hombre o mujer pueden verse expuestos y otra faceta interna, referida a la indefensión de las personas frente a los riesgos (falta de recursos con los que enfrentar esas situaciones). La vulnerabilidad, en su relación con el género ha contribuido a la identificación de dimensiones y aspectos clave de la desventaja social femenina. La crítica que se le ha hecho a este enfoque es su limitado alcance operacional, ya que aunque ha ayudado a los avances de políticas y al reconocimiento del reparto desigual de recursos, a su vez, ha confundido vulnerabilidad en el acceso a los recursos con fragilidad de la mujer (situando a las mujeres como víctimas pasivas para así legitimar las políticas asistencialistas).

- El enfoque de exclusión se plantea en la década de los noventa, y favorece una ampliación en la conceptualización y en el análisis de la desventaja social en el discurso internacional. Es un concepto relacional con dos rasgos principales: la carga semántica más marcada de la pobreza y la vulnerabilidad, y una significación de la exclusión como privación de derechos, siendo un concepto 
activo que conlleva responsabilidad externa y acción pública. Esta perspectiva de la exclusión ayuda a la conceptualización y al análisis de la desventaja social y presenta dos niveles de análisis, uno con carácter multidimensional (sociocultural y político) y un énfasis en los procesos dinámicos y multicausales (como complemento al entorno de los grupos, a los procesos y a las prácticas de exclusión). Estos procesos pueden ser macroeconómicos, institucionales, de actores sociales y espaciales.

- El enfoque de la desigualdad. Este enfoque explica cómo las personas satisfacen sus necesidades a través de una variedad de recursos además de los ingresos económicos, y cómo acceden a diferentes relaciones institucionales fuera del mercado. En términos de Amartya Sen (2010) en cuanto al ejercicio de derechos, las desigualdades son el resultado de dotaciones inadecuadas de recursos, donde estos se intercambian por otros o por prácticas reguladas donde no se contemplan las normas que garantizan el acceso a los derechos. El enfoque de las desigualdades plantea interrogantes de identidades e intereses así como sobre las divisiones de trabajo, poder y recursos. Se preocupa de una manera más dinámica de los procesos de exclusión y marginalización, lo que provoca cambios en la configuración de las relaciones.

Desde los movimientos de mujeres, se ha planteado la revisión desde este último enfoque, de las desigualdades codificadas en reglas y prácticas que diferentes áreas institucionales ejercen sobre los derechos de varones y mujeres, ya que este enfoque considera que la pobreza es un resultado de fallas en la obtención de derechos, que a su vez generan diferentes formas de privación (Kabeer, 1998; 2003). La desigualdad no existe en abstracto, surge siempre a partir de una definición sobre lo que comporta ser desigual en comparación con alguien. La desigualdad de género implica comparar al grupo privilegiado, los varones, con el desigual, es decir, las mujeres y partir de esta definición y visibilidad se han impulsado políticas públicas para la igualdad entre los géneros y se han movilizado organizaciones de mujeres cuyo resultado ha sido cambios significativos en la organización de género de la sociedad moderna (Gallegos, 2007).

Para corregir la desigualdad de las mujeres también es imprescindible mirar las dos caras de la vida social: los conflictos por los intereses contradictorios de los desiguales y la necesidad de elaborar consensos para cambiar la sociedad. Primero ha sido necesario darle legitimidad política al discurso de la desigualdad de las mujeres para que se tomen medidas para corregirlas. Pero esto ha mostrado en el camino que el problema no es solo de las mujeres, que se trata de un problema de estructura social que también se debe modificar (Astelarra, 2008; 2009). Por ello, a continuación, se van a sintetizar los principales marcos de referencia en los que se ha sustentado la implantación de los servicios sociales con perspectiva de género. 


\section{MARCOS DE REFERENCIA DE LA IMPLANTACIÓN DE LOS SERVICIOS SOCIALES: COHESIÓN SOCIAL, DESIGUALDAD, TRANSVERSALIDAD E INTERSECCIONALIDAD}

A partir de 1982 se manifestó en España una seria preocupación por el desarrollo de un modelo de política de servicios sociales personales que asumiera una perspectiva cercana a la estructura existente en los países europeos, con un Estado de bienestar consolidado. La clave del desarrollo de este nivel de políticas públicas se sustenta en el concepto de cohesión social.

La cohesión social se plantea como fin de las políticas públicas, con el objetivo de que todos los miembros de la sociedad se sientan parte activa de ella como contribuidores al progreso y como beneficiarios de este. En una inflexión histórica caracterizada por cambios profundos y veloces, precipitados por la globalización y por el nuevo paradigma de la sociedad de la información, se evidencia que las sociedades que ostentan mayores niveles de cohesión social brindan un mejor marco institucional para el crecimiento económico y la igualdad y estabilidad social (Ocampo, 2004). Pero era necesario un proceso de institucionalización que permitiera hacer realidad los planteamientos teóricos apuntados. Tres elementos van a permitir la aprobación de un marco legislativo de ámbito local que vehiculizara una mayor cohesión social, por una parte el espíritu descentralizador de la transición política, por otra el reconocimiento de la importancia de la territorialidad de la intervención y asimismo la perspectiva de la cercanía de los recursos a las demandas de la ciudadanía. En este marco tiene lugar la publicación de la Ley 7/1985, Reguladora de las Bases del Régimen Local, que a pesar de los límites a los que se ha hecho mención, permitió poner en marcha la propuesta de una red pública municipal de servicios sociales que diera respuesta a la cobertura de necesidades y a la integración social (Roldán, García y Nogués, 2013).

En 1988 se establece un plan nacional de apoyo al desarrollo de los servicios sociales públicos: el Plan Concertado de Prestaciones Básicas de Servicios Sociales en las Corporaciones Locales (1988), con objetivos ambiciosos en principio, que tuvo que adaptarse a dos realidades que rebajaron las expectativas de partida: la limitada aportación económica destinada en los presupuestos generales del Estado para tal fin y la necesidad de pactar ese plan con las comunidades autónomas competentes de forma exclusiva en la oferta y el desarrollo de los servicios sociales. Como no podía ser de otra forma, este plan se convirtió en pocos años en un elemento residual y han sido las comunidades autónomas y los propios municipios quienes han asumido la carga del desarrollo de las políticas de servicios sociales. Asimismo, a finales de los años ochenta, se implantan las rentas mínimas 
de inserción (RMI) en las CC.AA. y posteriormente los planes de inclusión social que han reforzado el papel de la Administración autonómica, al haber asumido en solitario el desarrollo de estos programas que han reforzado la limitada red de protección de los servicios sociales (Aguilar, 2010).

En diez años se llevó a cabo un desarrollo legislativo sin precedentes en todas las comunidades autónomas: las denominadas leyes de "primera generación" de servicios sociales que se iniciaron con la Ley vasca (1982) y finalizaron con la Ley foral navarra (1992), procurando un marco legal generalizado (Vilá, 2010). Estas normativas junto con la citada Ley Reguladora de las Bases del Régimen Local permitieron a corto plazo la implantación de estructuras específicas destinadas a desarrollar unas políticas de servicios sociales, que implicaban no solo elementos administrativos sino un cambio de mentalidad en lo que se refiere a la forma de afrontar las necesidades sociales. En este marco, una de las cuestiones que surgieron y que el pensamiento feminista incorporó a la agenda política fue un análisis y replanteamiento del tradicional papel la mujer como proveedora en la cobertura de las necesidades. En términos preestablecidos sobre las funciones tradicionalmente atribuidas a los géneros, las mujeres asumen: la función de proveedora de alimentación y ropa; la función asistencial respecto a las y los menores, las personas mayores, las personas enfermas y con discapacidad y la función relacional, en particular respecto a las funciones de socialización primaria y anticipatoria, etc. Todas estas funciones tienen en el ámbito doméstico y en el ámbito público, nombre femenino, tanto en el espacio de la actividad monetarizada, como en la no monetarizada. El cambio social y laboral experimentado por las mujeres durante el último período ha provocado una reformulación de las políticas de servicios sociales -que a pesar de su manifestación externa- se establece a partir del binomio mujer y actividad laboral; enfrentando dos ámbitos de actividad, el ámbito reproductivo versus el ámbito productivo (Del Olmo, 2014) que ponen en evidencia la situación de desigualdad entre mujeres y varones. Para combatir las desigualdades de género se fueron estableciendo distintas estrategias políticas, entre las que la igualdad de oportunidades ha sido la más extendida a escala internacional, incorporando mecanismos de acción positiva (Roldán, García y Nogués, 2013). Sin embargo, dichos mecanismos han demostrado también que son insuficientes para llegar al fondo de la cuestión: la escisión entre lo público y lo privado y la doble jornada que han de soportar las mujeres por haber accedido a la esfera pública. La igualdad de oportunidades, que apoya sobre todo la incorporación de la mujer a la esfera pública, ha logrado derechos formales pero no sustanciales, puesto que las mujeres se enfrentan con desventajas no solo familiares, sino también ideológicas y de poder (Carrasco, 2009; Pérez Orozco, 2010).

A nivel nacional se desarrollaron políticas de igualdad y su repercusión tuvo lugar en todos los niveles de las administraciones del Estado. En el ámbito 
local desde principios del 2000 se avanzaba en la superación de las actuaciones de carácter reivindicativo y de discriminación positiva ${ }^{7}$, con campañas de promoción de la mujer y de consolidación de nuevos valores tanto dentro del ámbito de lo público como de lo privado para progresar en la paridad de género (Roldán, 2004). En el nivel macrosistémico la evolución se ha caracterizado por la ampliación de las competencias del gobierno municipal y la aplicación de la transversalización de la perspectiva de género en todos los niveles de la política social. La transversalización de género ha sido definida poniendo el énfasis en dos perspectivas que se consideran complementarias: el efecto de las políticas sobre mujeres y varones, por un lado, y el propio proceso político institucional y de desarrollo de las capacidades necesarias para llevar a cabo esas políticas de igualdad de género, por otro. En último término, la estrategia de transversalización busca generar una mayor igualdad de género en nuestras sociedades. Pero qué duda cabe que para ello se requiere la transformación, reforma o reorganización de las instituciones y políticas, de modo que adquieran las capacidades necesarias para promover políticas con igualdad de género y que ellas mismas sean equitativas. Se trata, por tanto, de dos miradas o puntos de vista complementarios de la transversalización: desde las instituciones productoras y gestoras de políticas o desde el efecto de esas políticas sobre las mujeres y los varones que deben beneficiarse de los derechos y las oportunidades en condiciones de igualdad (López, Cirujano, Del Olmo, Sevilla y Sánchez, 2007).

Además del enfoque de la transversalidad, en el análisis de las políticas públicas con enfoque de género, es interesante rescatar también el concepto de interseccionalidad, que se puede plantear desde dos perspectivas que son importantes en este artículo: 1) La "interseccionalidad estructural", que se refiere a la experiencia directa que tienen las personas, cómo las intersecciones entre diferentes desigualdades pueden afectar estructuralmente a sus oportunidades económicas, políticas y sociales, creando desventajas para sujetos que se encuentran en el punto de intersección entre desigualdades concretas; 2) La "interseccionalidad política" que se refiere a la relevancia que las intersecciones entre desigualdades tienen para las estrategias políticas de instituciones y movimientos sociales, en el sentido de que las estrategias políticas que se dirigen a una desigualdad concreta por lo general no son neutrales respecto a las demás desigualdades, sino que pueden, por ejemplo, promover la igualdad de género y a la vez, discriminar a las mujeres inmigrantes, mayores u homosexuales (Lombardo y Verloo, 2010: 12).

\footnotetext{
${ }^{7}$ Aunque el término de discriminación positiva a día de hoy está en desuso, hemos querido reflejarlo tal y como se planteaba a principios del año 2000 (nota de las autoras).
} 
En este contexto, se produce lo que podemos llamar "la paradoja de género de la cohesión social": las mujeres son las principales proveedoras de cohesión social en términos de cuidados y formas de relación, a la vez que las principales excluidas de la cohesión social en términos de igualdad de oportunidades, ciudadanía y participación. Difícilmente podrá la política pública cumplir con su objetivo de cohesión social, garantizando que la ciudadanía pueda ejercer sus derechos fundamentales, si no se abordan las cuestiones de desigualdad de género que se manifiestan en la feminización de la pobreza, la violencia de género, la menor participación política de las mujeres, la desigualdad laboral y salarial, el analfabetismo femenino o la dificultad de estas para tener una salud sexual y reproductiva plena, entre otros problemas que claramente afectan a las mujeres (López, Cirujano, Del Olmo, Sevilla y Sánchez, 2007: 20-22). La complejidad de todas estas cuestiones requieren una modificación de las políticas de género, autoras como Bustelo y Lombardo (2007) apuntan que tanto los problemas que se abordan como las soluciones están dirigidos al sexo, es decir a las mujeres exclusivamente y no al género versus mujeres y varones y su relación. De este modo las mujeres son las que tienen el problema y a las que se les demandan cambios, mientras que los varones no se considera que formen parte del problema y no se les solicita cambios sustanciales.

Los marcos de referencia indicados han permitido ir estableciendo unas políticas de servicios sociales con perspectiva de género. Se ha avanzado significativamente en los últimos treinta años pero nos enfrentamos a un momento crítico en el que la crisis está permitiendo la toma de decisiones políticas que ponen en entredicho las conquistas alcanzadas y los referentes teóricos que las han sustentado (Pérez Orozco, 2006; 2010). Así la política local que ahora se plantea ofrece un incierto futuro a los servicios sociales municipales y a la perspectiva de género tal y como se desarrollará en los siguientes epígrafes.

\section{LA NUEVA LEGISLACIÓN Y SU IMPACTO EN LOS SERVICIOS SOCIALES Y EN LA PERSPECTIVA DE GÉNERO}

La nueva legislación de régimen local, la Ley 27/2013, de 27 de diciembre, de Racionalización y Sostenibilidad financiera de las Entidades Locales ha suscitado críticas, tanto desde el ámbito profesional, como del científico y académico que califican la actual reforma como mínimo de fallida (Almeida, 2013; Bosch y Solé-Ollé, 2014; Campus y Lago, 2014; Lapuente, 2014; García Herrero, Breznes, Barriga y Ramírez, 2014) como por parte de las comunidades autónomas que han presentado recursos, en este sentido, el Tribunal Constitucional ya ha admitido recursos presentados por los gobiernos de Andalucía, Cataluña, Canarias y Asturias, así como por los parlamentos de Extremadura, Cataluña, Andalucía, Navarra y por 130 diputados y diputadas de distintas fuerzas políticas. 
Los principales aspectos introducidos por la Ley y que se cuestionan en los textos señalados se pueden sintetizar en los siguientes:

- Intento de clarificación de las competencias de los municipios para evitar duplicidades de actuación con las de las Comunidades Autónomas (CC.AA.);

- Vaciado de competencias municipales que son transferidas a las Comunidades Autónomas y Diputaciones;

- Medidas encaminadas a favorecer la fusión voluntaria de municipios;

- Medidas destinadas a racionalizar la estructura de la Administración local, mejorar la transparencia y facilitar el control financiero;

- Medidas que limitan la discrecionalidad de los entes locales en términos de remuneración de cargos públicos;

- $\quad$ Efectos sobre el ahorro en la prestación de servicios públicos.

Dadas las limitaciones de este artículo, analizaremos los tres primeros puntos, por considerar que son los que tienen un mayor impacto en la política local para desarrollar luego los aspectos específicos relativos a los servicios sociales desde la perspectiva de género.

La pretensión de la Ley es clarificar las competencias locales mediante, por una parte, la transferencia de competencias en sanidad, educación y servicios sociales a las CC.AA y, por otra parte, con la prohibición del ejercicio de lo que denomina competencias impropias. Pero el problema en cuanto a las indicadas transferencias es que, al menos en el ámbito de los servicios sociales, los municipios se encuentran mejor situados que las CC.AA. para su prestación por su proximidad y capacidad de detección de necesidades de la ciudadanía. En lo que al ejercicio de competencias impropias se refiere, la realidad es que es el resultado de una presión social que se ejerce sobre el nivel de gobierno más próximo, por ello no parece que tenga mucho sentido prohibir la atención de demandas ciudadanas sin tener en cuenta cauces alternativos hacia otros niveles de gobierno (Campus y Lago, 2014).

- $\quad$ La Ley prevé que las competencias propias más importantes de los municipios sean transferidas a las diputaciones en el caso de los municipios de menos de 20.000 habitantes, cuando estos no puedan demostrar que la prestación del servicio se está haciendo de forma eficiente. En ese caso, se indica que la Diputación 'coordinará' la prestación de los servicios y que previo acuerdo con el municipio los servicios serán traspasados a la Diputación. En cualquier caso, establece la obligación por parte del municipio de demostrar que el 'coste efectivo' de la prestación del servicio es menor que el establecido por la Diputación. Las críticas al protagonismo otorgado a las diputaciones no se han hecho esperar 
(Ramió, 2013; Bosch y Solé-Olle, 2014). En cuanto al tema de la reducción de costes que puede suponer el traspaso a las diputaciones, parece no tener en cuenta que los costes unitarios de prestación no dependen tanto del número de personas usuarias como de su dispersión en el territorio. A lo que se une la falta de experiencia en este terreno de las diputaciones, y además, resulta paradójico dar relevancia en este momento a una estructura cuestionada por su escasa representación democrática, puesto que la elección es de forma indirecta por un procedimiento incomprensible para la mayoría de la población.

- Otro tema objeto de discusión es el referido a los incentivos a la fusión de municipios, abordado de forma muy tímida en la Ley, pues se considera que esos incentivos no van a ser efectivos y no van a conseguir que los municipios se fusionen. Al respecto, la apuesta ha sido de otra opción ya comentada: el refuerzo de las diputaciones. En algunos textos se apunta (Lapuente, 2014; Bosch y Solé-Olle, 2014) que se debería haber optado por una apuesta más decidida de las fusiones obligatorias con el objetivo de alcanzar municipios con un tamaño mínimo de 5.000 habitantes y en un futuro aproximarse a los 10.000. Los ejemplos de países como Dinamarca, Suecia, Francia o Alemania (Wollmann, 2008; Blom-Hansen, Houlberg y Serritzlew, 2013), podrían constituir un camino a seguir, ya que muestran que las amalgamaciones de municipios, racionalizan el mapa en entidades no solo de mayor tamaño medio, sino también de una mayor homogeneidad que permite una "sana" competitividad inter-municipal así como una mayor difusión de las "buenas prácticas" pero también se señala que esta opción no está exenta de problemas, al ser una opción estrictamente económica. Se apunta que "el aumento del tamaño de la jurisdicción hace posible un sistema político con menores costes económicos, sin embargo, puede haber un precio a pagar en términos de reducción del bienestar alcanzado y de escasez democrática" (Blom-Hansen, Houlberg y Serritzlew, 2014: 12).

Descendiendo al ámbito específico de los servicios sociales y la perspectiva de género, la Ley establece en su artículo 25.2.e) la responsabilidad de las entidades locales en materia de servicios sociales y las restringe a: "Evaluación e información de situaciones de necesidad y la atención inmediata a personas en situación o riesgo de exclusión social". Pero para completar el ámbito competencial de los municipios en materia de Servicios Sociales que se deduce de la mencionada Ley, será preciso definir también qué competencias deben asumir los municipios vía delegación.

Asimismo, la LRSAL establece en la modificación que realiza del artículo 27.3de la LRBRL, un catálogo de competencias que "con el objeto de evitar duplicidades administrativas, mejorar la transparencia de los servicios públicos y el servicio a la ciudadanía, y en general, contribuir a los procesos de racionalización administrativa, generando un ahorro neto de recursos, la Administración del Estado 
y las de las Comunidades Autónomas podrán delegar en los Ayuntamientos (...)" entre las que está la "Prestación de los servicios sociales, promoción de la igualdad de oportunidades y la prevención de la violencia contra la mujer" (art.27.3.c)). Dichas competencias se podrán delegar en la forma establecida en la Ley, es decir, que sea aceptada por el municipio y por un periodo estable temporal que no podrá ser inferior a cinco años; acompañada de la correspondiente dotación presupuestaria; con una cobertura del $100 \%$ del coste del servicio; y su cobro deberá estar garantizado. Si bien la Disposición Transitoria Segunda de la LRSAL prevé un periodo transitorio, hasta el 31 de diciembre del año 2015, para que la titularidad de las competencias propias municipales sobre prestación de servicios sociales y de promoción y reinserción social y la prestación de los servicios derivados de tales competencias sean asumidas por las Comunidades Autónomas, sin embargo, la aplicación de este periodo puede ser contradictoria, por lo que no debería ser aplicable, ya que ello significaría tanto como reconocer que la legislación sectorial autonómica no puede atribuir también competencias propias a los municipios en servicios sociales (Estudi Consultoria Sector Públic, 2014). De uno u otro modo lo que se alega es que prestar los servicios de proximidad desde las administraciones autonómicas sería claramente antieconómico e ineficaz (Fernández Muñoz, 2011; García Herrero, Breznes, Barriga y Ramírez, 2014).

Otro aspecto a tomar en consideración es la siguiente cuestión: ¿Son competencias propias de los municipios solo las establecidas en el artículo 25.2. de la LRSAL o también forma parte de este concepto las competencias reconocidas a los municipios por la legislación sectorial autonómica? (Estudi Consultoria Sector Públic, 2014). La respuesta a esta pregunta parece que ha despejado las posibles dudas, así la Federación Española de Municipios y Provincias (FEMP, 2014) se hace eco del criterio del Ministerio de Hacienda y Administraciones Públicas (MHAP, 2014), en relación con esta cuestión y considera que el listado de materias del artículo 25.2, sobre las que los municipios ejercerán en todo caso competencias propias, se establece "sin perjuicio de lo que puedan prever las leyes sectoriales estatales y autonómicas" (MHAP, 2014). Por tanto, cabe interpretar que los municipios pueden ejercer competencias propias sobre materias no incluidas en el artículo 25.2.e) si se las atribuyen las leyes sectoriales de servicios sociales, normativas en la que se hace referencia a una perspectiva amplia de los servicios sociales y a la incorporación de la perspectiva de género en los mismos.

Otra de las críticas al nuevo texto legal es la supresión del artículo 28 de la LBRL conforme al cual "los municipios pueden realizar actividades complementarias de las propias de otras administraciones públicas y, en particular, las relativas a la educación, la cultura, la promoción de la mujer, la vivienda, la sanidad y la protección del medio ambiente". Este artículo ha servido de fundamento jurídico para que ayuntamientos, mancomunidades, consorcios y 
diputaciones pudieran realizar la promoción de actividades, así como para la prestación de cuantos servicios públicos contribuyan a satisfacer las necesidades y aspiraciones de la ciudadanía en materia de género. La supresión de este artículo ha recibido grandes críticas por considerar que supone un menoscabo a actuaciones como la promoción de la mujer o la lucha contra la violencia de género que se consideran obligación de todas las administraciones públicas, por su transversalidad (Instituto Andaluz de la Mujer, 2013). En este terreno también se apunta la posible contradicción que puede suponer la aplicación del art. 22 de la Ley Orgánica 3/2007, de 22 de marzo, para la Igualdad Efectiva de Mujeres y Hombres (Almeida, 2013) que establece que: "con el fin de avanzar hacia un reparto equitativo de los tiempos entre mujeres y hombres, las corporaciones locales podrán establecer Planes Municipales de organización del tiempo de la ciudad. Sin perjuicio de las competencias de las Comunidades Autónomas, el Estado podrá prestar asistencia técnica para la elaboración de estos planes".

Todos los aspectos señalados entran en contradicción con lo que se aboga en el ámbito europeo que defiende y apoya los denominados servicios sociales de interés general cuyos objetivos, entre otros, se centren en servicios orientados a la persona, diseñados para responder a las necesidades humanas vitales, en especial las necesidades de personas vulnerables (Comisión Europea, 2004; 2006; 2007), aspecto este coincidente con lo establecido en el artículo 25 ya señalado de la LRSAL. Pero el marco europeo es más ambicioso en los objetivos que deben cumplir los servicios sociales y, así, apunta que desempeñan una función preventiva y de cohesión social y contribuyen a la no discriminación, a la igualdad de sexos, a la calidad de vida y a asegurar la creación de la igualdad de oportunidades para todos y todas. Por lo que esta perspectiva podría incorporarse como concreción de las competencias en servicios sociales vía delegación y de la perspectiva de género en la acción social municipal, de otro modo la nueva normativa local podría entrar en contradicción con las directrices europeas.

Tabla 2: Complejidad y contradicciones de las competencias en Servicios Sociales desde la perspectiva de género que plantea la LRSAL

\begin{tabular}{|l|l|}
\hline Marcos de referencia & Planteamientos competenciales \\
\hline $\begin{array}{l}\text { LRSAL: art. 25.2.e) } \\
\text { Responsabilidad de las entidades } \\
\text { locales en materia de } \\
\text { Servicios Sociales }\end{array}$ & $\begin{array}{c}\text { "Evaluación e información de situaciones de } \\
\text { necesidad y la atención inmediata a personas en } \\
\text { situación o riesgo de exclusión social" }\end{array}$ \\
\hline $\begin{array}{l}\text { LRSAL: art.27.3.c) } \\
\begin{array}{l}\text { La Administración del Estado y y } \\
\text { las de las Comunidades }\end{array}\end{array}$ & $\begin{array}{c}\text { "Prestación de los servicios sociales, promoción de } \\
\text { la igualdad de oportunidades y la prevención de } \\
\text { la violencia contra la mujer" }\end{array}$ \\
\hline
\end{tabular}




\begin{tabular}{|c|c|}
\hline $\begin{array}{l}\text { Autónomas podrán delegar } \\
\text { en los Ayuntamientos tales } \\
\text { como }\end{array}$ & \\
\hline $\begin{array}{l}\text { LRSAL: supresión del artículo } \\
28 \text { de la LBRL conforme al } \\
\text { cual: }\end{array}$ & $\begin{array}{l}\text { "Los municipios pueden realizar actividades } \\
\text { complementarias de las propias de otras } \\
\text { administraciones públicas y, en particular, las } \\
\text { relativas a la educación, la cultura, la promoción } \\
\text { de la mujer, la vivienda, la sanidad y la } \\
\text { protección del medio ambiente" }\end{array}$ \\
\hline $\begin{array}{l}\text { Leyes sectoriales de servicios } \\
\text { sociales de las } 17 \\
\text { Comunidades Autónomas }\end{array}$ & $\begin{array}{l}\text { Normativas en las que se hace referencia a una } \\
\text { perspectiva amplia de los servicios sociales y a } \\
\text { la incorporación de la perspectiva de género en } \\
\text { los mismos. }\end{array}$ \\
\hline $\begin{array}{l}\text { Ley Orgánica } 3 / 2007 \text {, de } 22 \text { de } \\
\text { marzo, para la Igualdad } \\
\text { Efectiva de Mujeres y } \\
\text { Hombres: art. } 22\end{array}$ & $\begin{array}{l}\text { "Con el fin de avanzar hacia un reparto equitativo } \\
\text { de los tiempos entre mujeres y hombres, las } \\
\text { corporaciones locales podrán establecer Planes } \\
\text { Municipales de organización del tiempo de la } \\
\text { ciudad. Sin perjuicio de las competencias de las } \\
\text { Comunidades Autónomas, el Estado podrá } \\
\text { prestar asistencia técnica para la elaboración de } \\
\text { estos planes." }\end{array}$ \\
\hline $\begin{array}{l}\text { COMISIÓN EUROPEA (2007): } \\
\text { Servicios de Interés general } \\
\text { incluidos los sociales: un } \\
\text { nuevo compromiso europeo. } \\
\text { COM (2007) }\end{array}$ & $\begin{array}{l}\text { Objetivos de los servicios sociales de interés } \\
\text { general: } \\
\text { servicios orientados a la persona, diseñados } \\
\text { para responder a las necesidades humanas } \\
\text { vitales, en especial las necesidades de personas } \\
\text { vulnerables. } \\
\text { desempeñan una función preventiva y de } \\
\text { cohesión social y contribuyen a la no } \\
\text { discriminación, a la igualdad de sexos, a la } \\
\text { calidad de vida y a asegurar la creación de la } \\
\text { igualdad de oportunidades para todos y todas }\end{array}$ \\
\hline
\end{tabular}

Fuente: elaboración propia

\section{POSIBILIDADES E INCERTIDUMBRES DEL FUTURO DE LOS SERVICIOS DE SOCIALES LOCALES Y DE LA PERSPECTIVA GÉNERO: ELEMENTOS PARA EL DEBATE}

La aprobación de esta nueva legislación no permite todavía un análisis pormenorizado de su aplicación y sus consecuencias. Sin embargo, se pueden vislumbrar diferentes opciones de cara al futuro, aspectos que están siendo objeto 
de tratamiento en diferentes textos académicos y profesionales a los que a lo largo de este artículo se han hecho referencia. Se van a apuntar, por tanto, los elementos fundamentales de la Ley que se han considerado en este artículo y las perspectivas, a veces coincidentes a veces contradictorias, que aparecen en los textos, lo que permite conformar a modo de conclusiones elementos para el debate acerca de posteriores decisiones sobre las que habrá que optar en aras de paliar las incidencias de esta reforma legislativa calificada como se ha podido observar como fallida, por contar con un texto normativo que se considera impreciso, contradictorio e incompleto. No obstante se pueden observar sombras, pero también luces que permitan perspectivas más halagüeñas. Las cinco cuestiones que se van a tratar, a modo de conclusiones, se centran en los siguientes aspectos: la autonomía local, el ámbito competencial, traspasos y delegaciones de competencias, la financiación y la perspectiva de género.

\subsection{Autonomía Local}

Existe un acuerdo expresado en distintas publicaciones sobre que la nueva legislación ha llevado a cabo una limitación o reducción de la autonomía municipal garantizada, cuyas consecuencias finales se advertirán en la medida en que las legislaciones sectoriales sigan expresamente sus pautas reductoras o, por el contrario, se inclinen por reconocer más poder político a los municipios, en cuyo caso será imprescindible un acompañamiento de financiación suficiente (Estudi Consultoria Sector Públic, 2014). Almeida (2013) apunta un pronóstico más claramente negativo al indicar una importante reducción cuantitativa del grado de autonomía local, en el campo de los servicios sociales, expresada en la reducción competencial, del nuevo art. 25.2 y "del ámbito material en el que, necesariamente, el legislador sectorial, autonómico o estatal, ha de conferir atribuciones a los ayuntamientos, la cual permitirá, por ejemplo, que las Comunidades Autónomas supriman algunas de las competencias que, actualmente, les reconocen en sus normas de régimen local o de servicios sociales" (Almeida, 2013:109).

\section{2 Ámbito Competencial}

En términos generales algunos de los escritos analizados plantean que el artículo 25.2 no es en sí mismo una norma que establece competencias a los municipios, sino un listado de materias sobre las que, posteriormente, legislaciones sectoriales podrán definir exactamente las competencias que tendrán los ayuntamientos. Asimismo se argumenta que la LRSAL no puede suponer una refundación absoluta del sistema de gobierno local en España, puesto que la disposición transitoria segunda de la LBRL, que no ha sido derogada por la LRSAL establece que mientras no se dicte legislación del Estado y de las Comunidades Autónomas según lo dispuesto en el artículo 25.2, los municipios conservarán las 
competencias que les atribuye la legislación sectorial vigente en la fecha de entrada en vigor de esta Ley. De otro modo sería dar por sentado que toda la legislación sectorial autonómica habría quedado en la práctica derogada por ese nuevo marco básico legislativo (Estudi Consultoria Sector Públic, 2014). Sin embargo, por una parte, la Federación Española de Municipios y Provincias en un informe elaborado al respecto(FEMP, 2014), plantea que el artículo 25.2 LBRL es un listado tasado y cerrado de competencias propias que tienen los municipios a partir de la entrada en vigor de la LRSAL, y por otra parte, se hace eco del criterio del Ministerio de Hacienda y Administraciones Públicas por el que se puede interpretar que los municipios pueden ejercer competencias propias sobre materias no incluidas en el artículo 25.2.e) si se las atribuyen las leyes sectoriales de servicios sociales. Parece que una amplitud de miras puede sobreponerse sobre la visión restrictiva que establece el referido artículo de la Ley.

En lo que se refiere específicamente a los servicios sociales el art 25.2.e) establece: "Evaluación e información de situaciones de necesidad social y la atención inmediata a personas en situación o riesgo de exclusión social", lo que plantea dos aspectos a tomar en consideración. Así, en primer lugar, se encuentra la referencia a "funciones en la evaluación e información de situaciones de necesidad social". Se podría deducir, tal y como apunta Almeida (2013) que hace referencia a que los ayuntamientos han de asumir competencias en relación con los servicios que aparecen en la clasificación establecida en el Catálogo de Referencia de Servicios Sociales, aprobado por Acuerdo del Consejo Territorial de Servicios Sociales y del Sistema para la Autonomía y Atención a la Dependencia del 16 de enero de 2013 (MSSSI, 2013), en dicho Catálogo, se clasifican como "servicios de información, orientación, asesoramiento y diagnóstico y se indica que son servicios que facilitan a las personas el acceso a los recursos, garantizan el acceso a otros sistemas de protección social y proporcionan apoyo en la realización de gestiones y tramitación de las diferentes prestaciones de servicios sociales y del sistema de protección social". Constituyen una tipología de servicios que se categorizan tanto como servicios generales o especializados. En una línea similar se expresa García Herrero (2014) al indicar que la competencia municipal en servicios sociales que establece el artículo 25 de la LRSAL debe interpretarse como la asunción de funciones tales como: información, orientación y asesoramiento; detección de situaciones de necesidad social; valoración y diagnóstico de situaciones de necesidad social; diseño de planes de intervención, prescripción de servicios y prestaciones económicas y técnicas; intervención profesional en los ámbitos individual, familiar, grupal y comunitario; atención inmediata a necesidades de subsistencia (García Herrero, 2014: 22). Estas lecturas de la legislación plantean también una continuidad con el modelo municipal de servicios sociales existente en la actualidad. 
En segundo lugar hay que tener en cuenta la mención a "la atención inmediata a personas en situación o riesgo de exclusión social", en este caso es necesario precisar el aspecto de "inmediatez", así como concretar los supuestos que se deben considerar como "situación o riesgo de exclusión social". En el primer caso parece pretender hacer referencia a la forma en la que las personas usuarias de los servicios sociales pueden acceder a ellos, de modo que la misma incluiría establecer servicios a los que se incorporarían directamente, por propia iniciativa o a través de una intervención social o administrativa (Almeida, 2013). La situación o riesgo de exclusión social, tal como indica Almeida, describe la posición en la que se encuentran determinados grupos de población especialmente vulnerables como: personas en situación de dependencia; víctimas de violencia de género; personas sin hogar; menores en situación de desprotección; personas inmigrantes; minorías étnicas, y personas con adicciones, etc. Como se puede observar, la perspectiva de género en este caso aparece especificada. Dicho autor concluye que siguiendo el indicado Catálogo de Referencia de servicios sociales, se podría defender que, al menos, los municipios han de tener competencias en: atención diurna y nocturna para personas sin hogar; intervención y orientación sociofamiliar; atención a menores en riesgo social y familiar; atención residencial (alojamiento de urgencia y para personas sin hogar) y atención de necesidades básicas. García Herrero (2014: 17), por su parte, ante la falta de definiciones legales de lo que se considera exclusión social, toma en consideración referentes legislativos tales como: la Ley 44/2007, de 13 de diciembre, para la regulación del régimen de las empresas de inserción en la que se especifica quiénes se consideran colectivos en riesgo o situación de exclusión social. Así como otra referencia normativa en la que se establece el "umbral de exclusión" como es el Real Decreto-Ley 6/2012 de medidas urgentes de protección de deudores hipotecarios sin recursos (modificado por Ley $1 / 2013$, de 14 de mayo, de medidas para reforzar la protección a los deudores hipotecarios, reestructuración de deuda y alquiler social). De uno u otro modo, una vez más estos autores defienden la necesidad de que los ayuntamientos cuenten con los servicios sociales que hasta ahora venían prestando.

\subsection{Traspaso Competencial y Delegación de Competencias}

Ya se ha indicado que la LRSAL ha optado por reducir, en el campo de los servicios sociales, los ámbitos donde los ayuntamientos han de ejercer, de forma necesaria, competencias propias. Pero al mismo tiempo ha reforzado la posibilidad de que tanto el Estado, como las Comunidades Autónomas, en este mismo sector, acuerden delegaciones de competencias propias de estas administraciones en los ayuntamientos. Así, según el nuevo art. 27 .3.c) es posible delegar en los municipios la prestación de los servicios sociales, de los servicios de promoción de la igualdad de oportunidades y de prevención de la violencia contra la mujer. Estas cuestiones afectan muy directamente a las políticas con perspectiva de género local, 
al dejar al arbitrio de la delegación de dichas actuaciones a los municipios en manos de otras administraciones.

Una vez más esta potenciación de las competencias delegadas son objeto de crítica, por cuanto limita la autonomía local, así el Congreso de Poderes Locales y Regionales del Consejo de Europa ha considerado que esta fórmula supone una visión reduccionista de la autonomía local establecida por la Carta Europea de Autonomía Local y ha advertido a España que las delegaciones de competencias a los municipios no son precisamente un medio de fortalecimiento de la autonomía local, sino todo lo contrario (Recomendación del Congreso de Poderes Locales y Regionales de 19 de marzo de 2013).

El análisis realizado por Estudi Consultoria Sector Públic (2014) considera que el traspaso a las CC.AA. de todos los servicios municipales en materia de servicios sociales que vayan más allá de lo previsto en el artículo 25.2.e), es de una gran complejidad práctica. Los servicios sociales que prestan los municipios son amplios en la mayoría de los casos y como se ha apuntado, se enmarcan en la legislación específica de las Comunidades Autónomas. Ya se ha indicado que se trata de un sector especialmente sensible en un marco de aguda crisis económica que está golpeando con especial fuerza a los segmentos más vulnerables y menos protegidos de la sociedad. Se crítica la complejidad que supondrá hacer efectivo el vaciamiento de competencias municipales a favor de las CC.AA. pues supondrá traspasos de servicios materiales y de personal de grandes dimensiones y además del ajuste financiero que tendrá lugar, supondrá redefinir por completo el modelo de servicios sociales con unos costes de transacción que más que el ahorro pretendido por la LRSAL pueden conllevar un crecimiento inusitado del gasto. $\mathrm{Y}$ todo para finalmente "delegar" la prestación de tales servicios en los municipios o en las diputaciones (Estudi Consultoria Sector Públic, 2014: 29).

Almeida, de igual modo, señala que la perspectiva anteriormente indicada parte de un análisis exclusivamente teórico, pero que si se cambia la perspectiva por un enfoque pragmático, los augurios pueden resultar más positivos. El autor establece dos premisas que le llevarán a esta conclusión. Por una parte, el hecho de que las competencias relacionadas con los servicios sociales, que podrían quedar en manos de las Comunidades Autónomas, constituyen, como se viene indicando, un sector muy sensible para la ciudadanía y de gran importancia en el mundo local, por lo que los municipios, con relativa seguridad, estarán deseosos de recuperarlas, sobre todo si, tal y como exige la Ley tendrán que estar acompañadas de una financiación suficiente. $\mathrm{Y}$, por otra parte, en el presente contexto de crisis económica, es probable que, a su vez, las Comunidades Autónomas tengan también un marcado interés en desprenderse de la gestión de las competencias indicadas, porque, entre otras razones, el propio proceso de asunción del ejercicio de estas 
funciones, hasta el momento municipales, implica unos importantes costes (Almeida, 2013: 113).

En el análisis realizado por García Herrero (2014) se toma en consideración que deben ser delegados a los municipios, con la correspondiente financiación de su coste efectivo, como la nueva Ley establece, todos los servicios de proximidad que no estén ya contemplados como competencia, lo que significa seguir asumiendo y completando el resto de prestaciones y servicios que configuran los hoy día denominados servicios de atención primaria o servicios sociales generales.

Es de esperar que la sensatez y el sentido común impere y este proceso que se anuncia quede definitivamente truncado, pues en un contexto tan duro de crisis económica que estamos viviendo, los servicios sociales de carácter municipal son, como también se apuntaba antes, uno de los pilares fundamentales de cohesión social, en cuanto que palía o mejora una situación social que puede derivar en un contexto explosivo si no se adoptan medidas para su contención y corrección.

En estos momentos las CC.AA. han iniciado la aprobación de legislaciones para adaptarse a la reforma local que plantea la LRSAL. Entre otras se señalan:

El "DECRETO LEY 4/2014, de 22 de julio, por el que se establecen medidas urgentes para adaptar los convenios, los acuerdos y los instrumentos de cooperación suscritos entre la Administración de la Generalidad y los entes locales de Cataluña a la disposición adicional novena de la Ley 27/2013, de 27 de diciembre, de racionalización y sostenibilidad de la Administración local" y la "LEY 1/2014, de 25 de julio, de Adaptación del Régimen Local de la Comunidad de Madrid a la Ley 27/2013, de 27 de diciembre, de Racionalización y Sostenibilidad de la Administración Local". Habrá que esperar a la aplicación de las diversas normativas autonómicas al respecto para conocer el alcance de las modificaciones que se van a producir en el sistema de servicios sociales.

\subsection{Financiación}

La LRSAL establece que cada Administración debe tener la responsabilidad y la capacidad de financiar por sí misma lo que la Ley establece que son competencias propias. Por eso este nivel competencial de los municipios en materia de servicios sociales, debe ser tenido en cuenta para determinar su sistema ordinario de financiación, estableciendo los indicadores mínimos de suficiencia financiera de la red local, que garanticen la equidad territorial, teniendo en cuenta las diversas variables que inciden en el coste de los servicios, tales como la dispersión de población (García Herrero, 2014). Sin embargo en la LRSAL se echa en falta una 
mayor regulación de la tramitación de la traslación de las competencias de los municipios a las administraciones autonómicas, debido en gran medida, a que considera que esa responsabilidad debe ser de las leyes reguladoras del sistema de financiación de las Comunidades Autónomas y de las haciendas locales, por ello a la vista de las actuales normas reguladoras del sistema de financiación de las Comunidades Autónomas y de las haciendas locales, cabe dudar que las mismas puedan satisfacer, de forma adecuada, el cometido que la LRSAL les encomienda. El problema radica en que esta reforma local se ha elaborado antes de modificar el actual sistema de financiación municipal, de manera que no se han evaluado ni contemplado las necesidades de financiación que conlleva este nivel de competencia municipal en servicios sociales. En consecuencia, parece que es imprescindible una reforma urgente de la legislación de la financiación indicada, para que las atribuciones en materia de servicios sociales, a las que atañe la disposición citada, puedan traspasarse en los plazos fijados.

Sin duda la cuestión financiera es clave en el mantenimiento del sistema de servicios sociales. Los datos del siguiente cuadro dan cuenta de la pérdida del apoyo financiero que los servicios sociales están experimentando:

Tabla 3:Gasto consolidado en Servicios Sociales y promoción social (miles de euros)

\begin{tabular}{|l|l|l|l|}
\hline $\mathbf{2 0 1 0}$ & $\mathbf{2 0 1 1}$ & $\mathbf{2 0 1 2}$ & $\mathbf{2 0 1 3}$ \\
\hline 6.246 .065 & 6.135 .042 & 5.872 .514 & 5.194 .164 \\
\hline
\end{tabular}

Fuente: Elaboración propia a partir de MINHAP ${ }^{8}$.

En cuatro años, en una situación de crisis aguda, el gasto ha descendido en más mil millones de euros, lo que pone en evidencia la falta de apoyo, de decisión y de inclusión en la agenda política de unos servicios que cumplen una función esencial de cohesión social.

\subsection{Género}

8 Ministerio de Hacienda y Administraciones Públicas. Secretaría de Estado de Administraciones Públicas. Secretaría General de Coordinación Autonómica y Local. Oficina virtual para la coordinación financiera con las Entidades Locales. Recuperado de: http://servicioswebbis.meh.es/apps/entidadeslocales/ (Junio, 10, 2014). 
A modo de cierre, y reflexionando sobre estos aspectos desde el enfoque de género, hay que señalar que de forma general a lo largo del texto se han ido indicando las inquietudes que, en materia de servicios sociales, crea el contenido del artículo 27.3.c) que muestra que "la Administración del Estado y las de las Comunidades Autónomas podrán delegar, siguiendo criterios homogéneos, entre otras, las siguientes competencias: Prestación de los servicios sociales, promoción de la igualdad de oportunidades y la prevención de la violencia contra la mujer". En lo que se refiere a la perspectiva de género, el amplio desarrollo de la misma ha tenido a nivel local y en concreto en el ámbito de los servicios sociales, así como los aspectos preventivos en una cuestión tan grave como es la violencia contra las mujeres se pueden ver gravemente afectadas si ambos temas no se incorporan como prioridad en la agenda política de las CC.AA. con un enfoque de actuaciones de proximidad que tan solo pueden ser desarrolladas desde los municipios por su cercanía con la ciudadanía. Paradójicamente esta situación puede llevar a romper con el principio fundamental de proximidad en la prestación de servicios sociales que rige en todo el ámbito europeo, asimismo puede poner en entredicho el principio de cohesión social en cuanto a que sectores vulnerables o en condiciones de desigualdad pueden quedar sin cobertura de los servicios sociales y afectar especialmente al principio de igualdad de género dada la feminización de los mencionados como sectores vulnerables.

\section{BIBLIOGRAFÍA}

ADELANTADO, José y JIMÉNEZ, Aida (2003): Las políticas de servicios sociales en las Comunidades Autónomas en Gallego, R.; Gomà, R. y Subirats, J. (eds.), Estado de Bienestar y Comunidades Autónomas. La descentralización de las políticas sociales en España. Madrid: Tecnos.

AGUILAR, Manuel (2010): La huella de la beneficencia en los Servicios Sociales. Zerbitzuan: Gizarte zerbitzuetarako aldizkaria. Revista de servicios sociales, 48, 9-16.

GÓMEZ ALGUACIL, Julio (2012): La quiebra del incompleto sistema de servicios sociales en España. Cuadernos de Trabajo Social, 25 (1), 63-74.

ALMEIDA, Marcos (2013): El incierto futuro de los servicios sociales municipales. Anuario de Derecho Municipal, 7, 93-144. 
ANTÓN, Antonio (2012): Política social en tiempos de crisis. Cuadernos de Trabajo Social, 25 (1), 49-62.

ASTELARRA, Judith (2008): Género y empleo. Documento de Trabajo, 32. Madrid: Fundación Carolina-CeALCI.

ASTELARRA, Judith (2009): Pacto entre géneros y políticas públicas: género y cohesión social. Debates, 50. Madrid: Instituto de la Mujer.

BLOM-HANSEN, Jens; HOULBERG, Kurt and SERRITZLEW, Soren (2014): Size, Democracy, and the Economic Costs of Running the Political System. American Journal of Political Science. DOI: 10.1111/ajps.12096

BOSCH, Nuria y SOLÉ-OLLÉ, Albert (2014): "La fallida reforma de la Administración Local" Reforma de la Administración Local en España, en Informe IEB Sobre Federalismo Fiscal y Finanzas Públicas, 13, 4-8. Recuperado de http://www.ieb.ub.edu/es/2012022056/federalismofiscal/federalisme-fiscal\#.U611IIdZpaQ (Junio, 15, 2014).

BUSTELO, María y LOMBARDO, Emanuela (2007): Políticas de igualdad en España y en Europa. Madrid: Cátedra.

CAMPUS, Concepción y LAGO, Santiago (2014): "La necesaria (y fallida) reforma de la Administración local" Reforma de la Administración Local en España, en Informe IEB Sobre Federalismo Fiscal y Finanzas Públicas, 13, 9-11. Recuperado de http://www.ieb.ub.edu/es/2012022056/federalismofiscal/federalisme-fiscal\#.U611IIdZpaQ (Junio, 15, 2014).

CARRASCO, Cristina (2009): Mujeres, sostenibilidad y deuda social. Revista de Educación, número extraordinario, 169-191.

CLERT, Carine (1998): De la vulnerabilidad a la exclusión: género y conceptos de desventaja social. ISIS Internacional, Ed. De las mujeres, 26, 42-58.

COMISIÓN EUROPEA (2004): Libro Blanco sobre los servicios de interés general. COM (2004) 374 final 12.05.2004, Bruselas.

COMISIÓN EUROPEA (2006): Aplicación del programa comunitario de Lisboa. Servicios sociales de Interés general en la Unión Europea. COM (2006) 177 final, 26.04.2006, Bruselas.

COMISIÓN EUROPEA (2007): Servicios de Interés general incluidos los sociales: un nuevo compromiso europeo. COM (2007) 724 final, 20.11.2007, Bruselas.

DEL OLMO, Nuria (2014): Servicios Sociales: la dimensión institucional de la perspectiva de género. Portularia, XIV (1), 107-117.

ESTUDI CONSULTORIA SECTOR PÚBLIC (2014): "Vademécum” sobre la ley de racionalización y sostenibilidad de la Administración local. Barcelona: Federació de Municipies de Catalunya. Recuperado de: http://www.dival.es/sites/default/files/oficina-de-informacion/VADEMECUMCOMPETENCIAS MUNICIPALES.pdf (Agosto, 12, 2014).

FEDERACIÓN ESPAÑOLA DE MUNICIPIOS Y PROVINCIAS (FEMP) (2014): Las competencias de los ayuntamientos en materia de servicios sociales tras la entrada en vigor de la Ley 27/2013 de racionalización y sostenibilidad de la Administración local. Recuperado de: http://www.femp.es/files/3580-865- 
fichero/Informe $\% 20$ competencias $\% 20$ en $\% 20$ materia $\% 20$ de $\% 20$ cooperación $\%$ 20al\%20desarrollo.pdf (Julio, 20, 2014).

FERNÁNDEZ MUÑOZ, Jesús Norberto (2011): Servicios Sociales, derechos de ciudadanía y atención centrada en la persona. TS nova: trabajo social y servicios sociales, 3, 37-48.

GALLEGOS, $\mathrm{M}^{\mathrm{a}}$ Teresa (2007): Equidad de género, política y cohesión social, en Cohesión social en Iberoamérica: algunas asignaturas pendientes. Revista de Pensamiento Iberoamericano, 1, 157-176. Madrid: CEALCI.

GARCÍA HERRERO, Gustavo (coord.), BREZNES, Ma Jesús, BARRIGA, Luis A. y RAMÍREZ, José Manuel (2014): Contenidos de la competencia municipal en servicios sociales, en el nuevo marco legal. Asociación Estatal de Directores y Gerentes en Servicios Sociales. Recuperado de http://www.directoressociales.com/images/documentos/novedaddiscusion/com petencia\%20local.lrsal\%204\%201\%201.pdf (Mayo, 15, 2014).

INSTITUTO ANDALUZ DE LA MUJER (2013): Informe relativo al impacto en las políticas de igualdad del anteproyecto de ley para la racionalización y sostenibilidad de la Administración Local. Consejo Andaluz de Participación de las

Mujeres.

Recuperadode:http://www.forumpoliticafeminista.org/sites/default/files/inform e\%20anteproyecto\%20reforma\%201ocal\%20maquetado_1.pdf (Agosto, 11, 2014).

KABEER, Nayla (1998): Tácticas y compromisos: nexos entre género y pobreza. ISIS Internacional, Ed. De las mujeres, 26, 19-24.

KABEER, Nayla (2003): Gender Mainstreaming in Poverty Eradication and the Millennium Development Goals. A Handbook for Policy-makers and Other Stakeholders. Commonwealth Secretariat, IDRC, CIDA. Recuperado de: http://www.idrc.ca/openebooks/067-5/ (Julio, 12, 2014).

LAPUENTE, Víctor (2014): "Dos pesos de la historia" Reforma de la Administración Local en España, en Informe IEB Sobre Federalismo Fiscal y Finanzas Públicas, 13, 12-16. $\quad$ Recuperado de http://www.ieb.ub.edu/es/2012022056/federalismo-fiscal/federalismefiscal\#.U611IIdZpaQ (Junio, 15, 2014).

LOMBARDO, Emanuela y VERLOO, Mieke (2010): La 'interseccionalidad' del género con otras desigualdades en la política de la Unión Europea. Revista Española de Ciencia Política, 23, 11-30.

LÓPEZ, Irene (coord.); CIRUJANO, Paula; DEL OLMO, Alicia; SEVILLA, Beatriz y SÁNCHEZ, Rocío (2007): Género y políticas de cohesión social. Conceptos y experiencias de transversalización. Madrid: FIAPP.

MINISTERIO DE HACIENDA Y ADMINISTRACIONES PÚBLICAS (MHAP) (5 de marzo de 2014): "Nota explicativa sobre la Ley 27/2013, de 27 de diciembre, de racionalización y sostenibilidad de la Administración Local". Recuperadode:http://www.minhap.gob.es/Documentacion/Publico/PortalVarios /Gesti\%C3\%B3n $\% 20 \mathrm{del} \% 20$ Portal/Nota $\% 20$ explicativa $\% 20 \mathrm{de} \% 201 \mathrm{a} \% 20$ refor ma\%20local.pdf (Julio, 2, 2014). 
MINISTERIO DE SANIDAD, SERVICIOS SOCIALES E IGUALDAD (MSSSI) (2013): Catálogo de Referencia de Servicios Sociales. Aprobado por el Consejo Territorial de Servicios Sociales y del Sistema para la Autonomía y Atención a la Dependencia. Recuperado de: https://www.msssi.gob.es//ssi/familiasInfancia/inclusionSocial/serviciosSociale s/Catalogo_Servicios Sociales/docs/CatalogoServiciosSociales.pdf (Julio, 25, 2014).

OCAMPO, José Antonio (2004): Reconstruir el futuro. Globalización, desarrollo y democracia en América Latina. Bogotá, D.C.: Comisión Económica para América Latina y el Caribe (CEPAL)/Grupo Editorial Norma.

OECD (2014): Spain: From Administrative Reform to Continuous Improvement. OECD Public Governance Reviews. OECD Publishing. Recuperado de: http://www.oecd-ilibrary.org/governance/spain-from-administrative-reform-tocontinuous-improvement 9789264210592-en (Julio, 13, 2014).

PÉREZ OROZCO, Amaia (2006): Amenaza tormenta: la crisis de los cuidados y la reorganización del sistema económico. Revista de Economía Crítica, 5, 7-37.

PÉREZ OROZCO, Amaia (2010): Crisis multidimensional y sostenibilidad de la vida. Investigaciones Feministas, 1, 29-53.

RAMIÓ, Carles (2013): Balance del impacto de las crisis económica, politica, social e institucional sobre las administraciones públicas en España 2011-2013: Diagnóstico y propuestas. GIGAPP Estudios/Working Papers, Programa de Doctorado en Gobierno y Administración y Pública. Madrid: Instituto Universitario de Investigación Ortega y Gasset. Recuperado de: http://www.gigapp.org/es/component/jresearch/?view=publication\&task=show \&id=937\#.U -WPPmSzQg (Agosto, 12, 2014).

RODRÍGUEZ CABRERO, Gregorio (2004a): Protección social de la dependencia en España. Documento de trabajo 44/2004. Madrid: Fundación Alternativas.

RODRÍGUEZ CABRERO, G (2004b): El Estado de Bienestar en España: debates, desarrollo y retos. Madrid: Fundamentos.

ROLDÁN, Elena (ed.) (2004): Género, politicas locales e intervención social. Análisis de los servicios de bienestar social municipal para la población femenina. Madrid: Editorial Complutense.

ROLDÁN, Elena y GARCÍA, Teresa (2006): Politicas de Servicios Sociales. Madrid: Síntesis.

ROLDÁN, Elena, GARCÍA, Teresa y NOGUÉS, Luis (2013): Los Servicios Sociales en España. Madrid: Síntesis.

SANTAMARINA, Cristina; MENDIGUREN, Verónica; MONDINE, Santiago y ROLDÁN, Elena (1999): Iniciativa Privada y Servicios Sociales. Madrid: Ministerio de Trabajo y Asuntos Sociales. Instituto de Migraciones y Servicios Sociales.

SEN, Amartya (2010): Desarrollo y Libertad. Buenos Aires: Planeta.

SUBIRATS, Joan (dir.) (2007): Los servicios sociales de atención primaria ante el cambio social. Madrid: Ministerio de Trabajo y Asuntos Sociales. 
VILÁ, Antoni (2010): Los cambios legislativos en materia de servicios sociales (2000-2009). En D. Casado (coord.) Leyes de servicios sociales del siglo XXI (pp.17-48). Madrid: Fundación Foessa.

WOLLMANN, Hellmut (2008): Comparing Local Government Reforms in England, Sweden, France and Germany. Recuperado de: www.wuestenrotstiftung.de/download/local-government (Agosto, 18, 2014). 